Editorial

\title{
Recent Advances in Solution Methods for Nonlinear Evolution Equations, Fluid Flow, and Heat and Mass Transfer
}

\author{
Sandile Motsa, ${ }^{1}$ Stanford Shateyi, ${ }^{2}$ Robert A. van Gorder, ${ }^{3}$ \\ Najib Laraqi, ${ }^{4}$ and B. Rush Kumar ${ }^{5}$ \\ ${ }^{1}$ School of Mathematics, Statistics and Computer Science, University of KwaZulu-Natal, Private Bag X01, Scottsville, \\ Pietermaritzburg 3029, South Africa \\ ${ }^{2}$ Department of Mathematics and Applied Mathematics, University of Venda, Private Bag X5050, Thohoyandou 0950, South Africa \\ ${ }^{3}$ Department of Mathematics, University of Central Florida, Orlando, FL 32816, USA \\ ${ }^{4}$ Laboratoire Thermique Interfaces Environnement, Université Paris Ouest, 50 rue de Sèvres, 92410 Ville d’Avray, France \\ ${ }^{5}$ Fluid Dynamics Division, School of Advanced Sciences, VIT University, Tamil Nadu, India
}

Correspondence should be addressed to Sandile Motsa; sandilemotsa@gmail.com

Received 12 August 2015; Accepted 12 August 2015

Copyright (C) 2015 Sandile Motsa et al. This is an open access article distributed under the Creative Commons Attribution License, which permits unrestricted use, distribution, and reproduction in any medium, provided the original work is properly cited.

The development of new methods for the solution of nonlinear ordinary and partial differential equations and many other types of equations that model real-life nonlinear phenomena continues to be an active area of research. In recent years, there has been an upsurge in research that is focused on developing improvements of existing solution techniques with the aim of optimising their efficiency and performance. Focus has also been directed to the development of solution methods that are sufficiently robust to solve complex problems that cannot be completely resolved using standard solution methods. This special issue aims to report on the recently developed solution methods and their application in solving real-life problems. The special issue contains articles drawn from a wide range of mostly engineering applications.

"On the Optimal Auxiliary Linear Operator for the Spectral Homotopy Analysis Method Solution of Nonlinear Ordinary Differential Equations" by S. S. Motsa seeks to identify the optimal linear operator for the solution of nonlinear ordinary differential equations. The linear operators can be used with different variants of the homotopy analysis method. The study introduces new procedures of defining the auxiliary linear operators and compares solutions generated using the new linear operators against solutions obtained using well-known linear operators. "Local Fractional Fourier Series Method for Solving Nonlinear Equations with Local
Fractional Operators" by Y.-J. Yang and S.-Q. Wang presents an implementation of the local fractional Fourier series method in solving nonlinear equations drawn from the fractal set. Examples discussed in the study of Y.-J. Yang and S.-Q. Wang include a local fractional differential equation, an integral-differential equation, and an integral-differential equation system. The approach used in the study is aimed at generalising and enriching the well-known local fractional Fourier series method. The generalized approach is reported to have the benefit of simplifying the process of solving partial differential or integral-differential equations. The simplification involves transforming the given equations into a system of ordinary differential equations, thereby reducing potentially complicated calculations to a relatively manageable computational task.

A new fourth-order energy conservative compact finite difference scheme is proposed by S. Li et al. in "A FourthOrder Conservative Compact Finite Difference Scheme for the Generalized RLW Equation." The proposed method is used to generate solutions of the generalized regularized long wave (GRLW) equation. Rigorous convergence and error analysis is presented to give insight into the numerical properties of the method from a theoretical viewpoint. The theoretical results are validated using numerical computational results for the solution of the GRLW for single solitary 
waves. Results from the numerical experimentation agree with the theory and demonstrate that the proposed method is accurate, effective, and reliable.

"Self-Similar Unsteady Flow of a Sisko Fluid in a Cylindrical Tube Undergoing Translation" by M. Khan et al. considers the unidirectional flow of a Sisko fluid in a cylindrical tube due to translation of the tube wall. Lie symmetry analysis is used to obtain similarity equations in the form of nonlinear ordinary differential equation (ODE). The ODE is numerically integrated using some packages that are available as built-in functions in the Matlab computing environment. The study gives new insights into the physics of the Sisko fluid and Newtonian flow. "Analytical Modelling of Three-Dimensional Squeezing Nanofluid Flow in a Rotating Channel on a Lower Stretching Porous Wall" by N. Freidoonimehr et al. employs the differential transform method (DTM) to study the threedimensional flow of a nanofluid in a rotating channel on a lower permeable stretching porous wall. The physical effect of different type of nanoparticles is studied using water as a based fluid. The effect of the pertinent physical parameters is illustrated graphically and important conclusion on the influence of these parameters on the flow profile is discussed.

In "On a Coupled System of Shallow Water Equations Admitting Travelling Wave Solutions," D. Burini et al. develop a nonlocal spectral formulation for three classic fluid equations. The study considers three inviscid, incompressible, irrotational fluids that are contained between the rigid walls and separated by two free interfaces. The study yields two coupled shallow water equations which have been studied numerically in order to prove the existence of solitary waves. The behaviour of the shallow wave equations is also analysed through graphical results. "Diffusion of Chemically Reactive Species in Casson Fluid Flow over an Unsteady Stretching Surface in Porous Medium in the Presence of a Magnetic Field" by G. Makanda et al. investigates the effects of heat transfer, magnetism, and porosity on Casson fluid flow. The governing differential equations are integrated using the Runge-Kutta-Fehlberg method.

"The Application of MPC in Microwave Heating Process Based on Model Constructed by Lambert's Law Combined with Temperature" by J. Li et al. compares Lambert's law with Maxwell's equation using the model predictive control (MPC) algorithm. "An Extended Finite Element Model for Fluid Flow in Fractured Porous Media" by F. Liu et al. proposes the extended finite element method numerical approach for investigating fluid flow in fractured porous media. The study highlights the benefits of partitioning the domain as a nonmatching grid without considering the presence of fractures. This allows for the easy treatment of arbitrarily multiple, kinking, branching, and intersecting fractures.

A new numerical algorithm based on the Smoothed Particle Hydrodynamics (SPH) method is proposed by X.J. Ma and M. Geni for the solution of fluid-solid coupling and complex free surface problems in "Simulation of Droplet Impacting on Elastic Solid with the SPH Method." The numerical simulations performed in the study reveal that the improved SPH method is able to unravel detailed information about the microdeformation of solid surfaces. The study also discusses the influence of the elastic modulus of solids on the impacting processes.

"Analytical Model of Fluid Flow through Closed Structures for Vacuum Tube Systems" by C.-H. Park et al. develops an analytical model to evaluate airtightness in vacuum tube structures. The model considered is based on anticipating that the pressure inside closed structures initially decreases and then rises with time. This is achieved by using Darcy's law and solving differential equations that consider the air permeability of the material and physical configuration of the tube.

"A Theorem for Finding Maximum Temperature in Wet Grinding” by J. L. González-Santander and G. Martín is a theoretical approach that analyses the solutions found in the literature for heat transfer in surface grinding. The study presents several theorems for the identification of the location of the maximum temperature in the different regimes of the work piece surfaces. The theoretical results presented in the study would be useful in avoiding thermal damage in surface wet grinding.

"Exact Solitary Wave and Periodic Wave Solutions of a Class of Higher-Order Nonlinear Wave Equations" by L. Zhang and M. Khalique considers the exact traveling wave solutions of a general fifth-order nonlinear wave equation and a generalized sixth-order $\mathrm{KdV}$ equation. As examples, solitary wave and periodic wave solutions of the Lax equation, the Ito equation, and a general sixth-order $\mathrm{KdV}$ equation are derived and simulated.

In summary, a number of recently developed solution methods have been proposed in this special issue. Other methods were introduced to explain phenomena that cannot be unravelled using standard methods.

\author{
Sandile Motsa \\ Stanford Shateyi \\ Robert A. van Gorder \\ Najib Laraqi \\ B. Rush Kumar
}




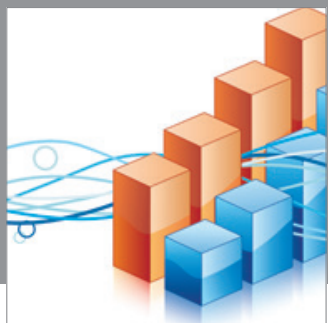

Advances in

Operations Research

mansans

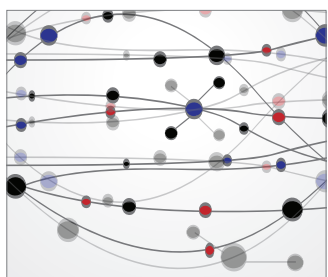

The Scientific World Journal
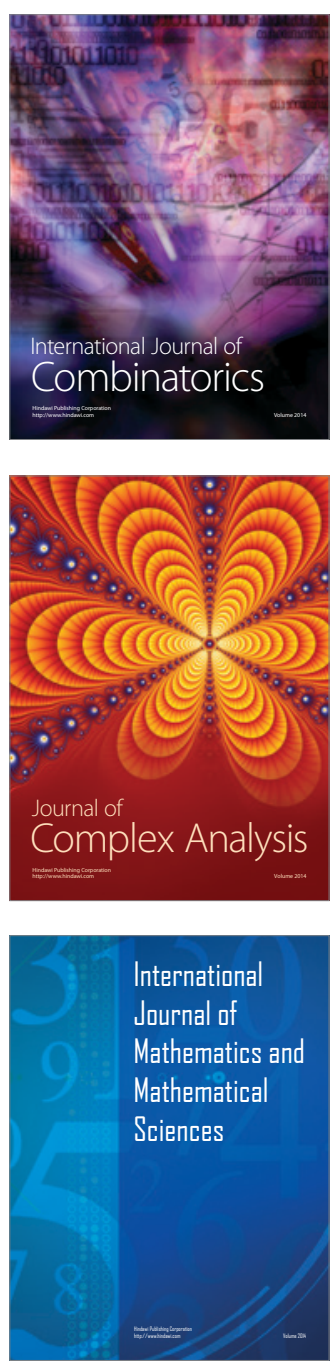
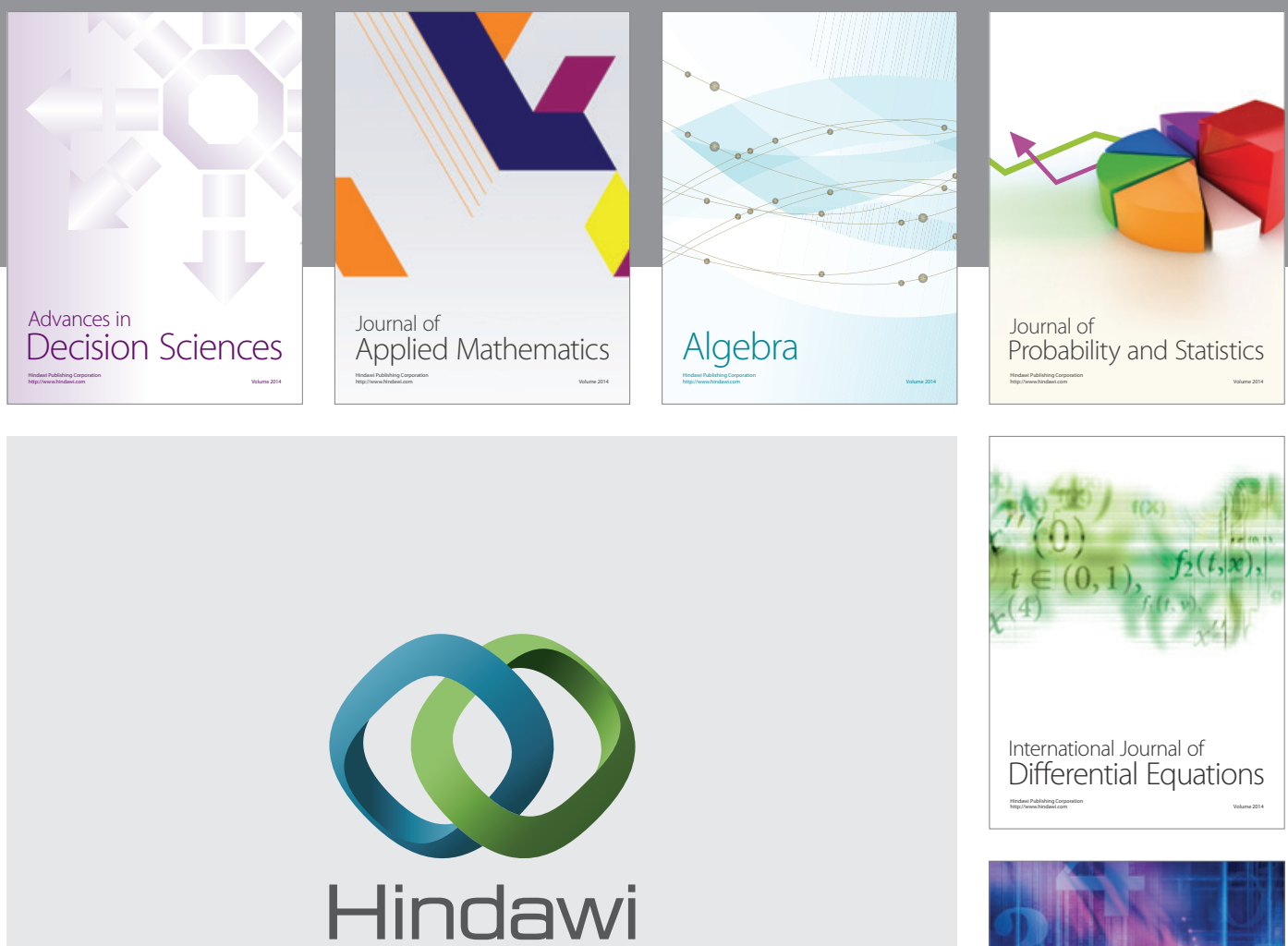

Submit your manuscripts at http://www.hindawi.com
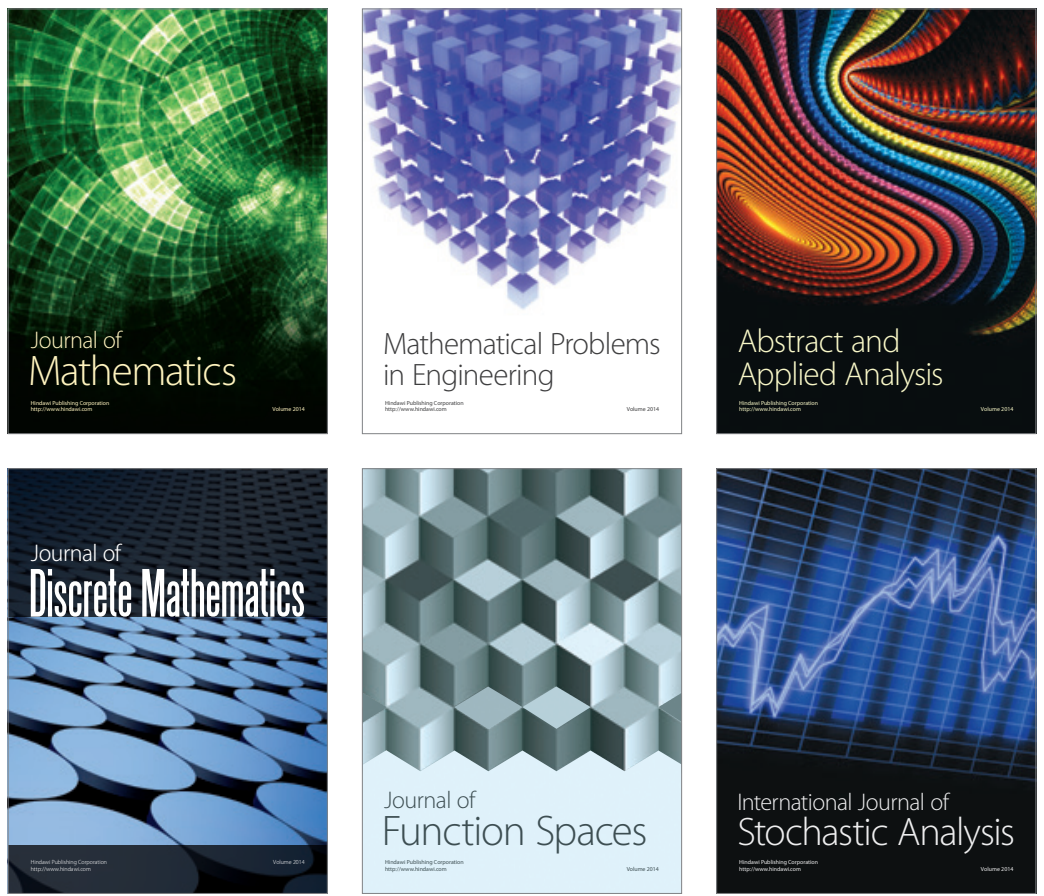

Journal of

Function Spaces

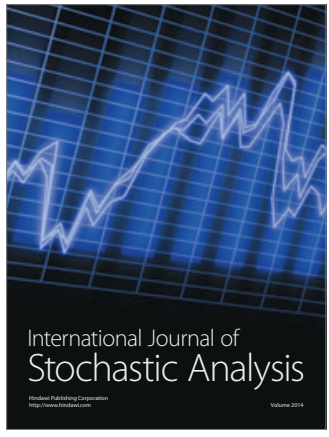

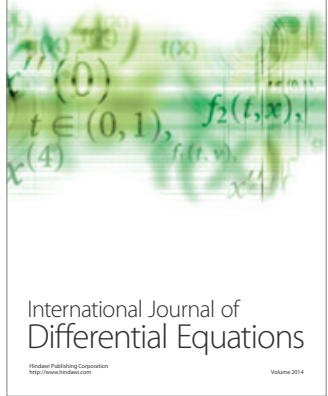
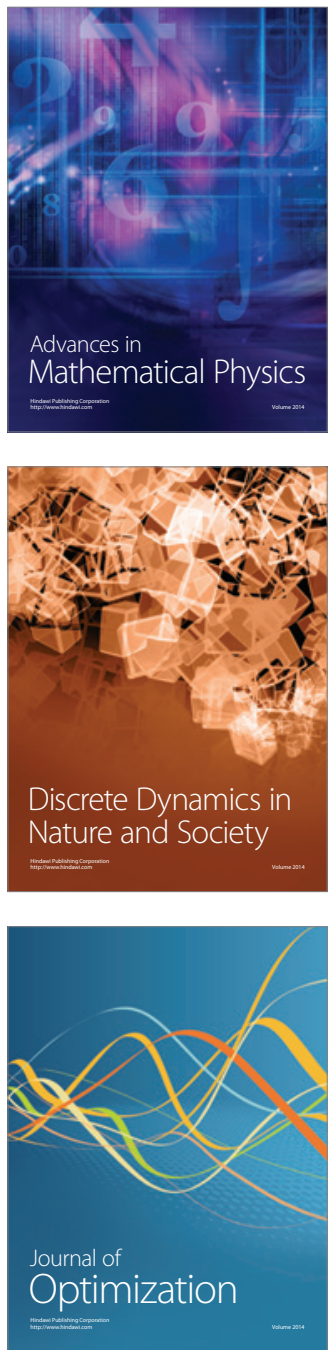